\title{
LINEAR PROGRAMMING AND PLASTIC LIMIT ANALYSIS OF STRUCTURES*
}

\author{
BY \\ W. S. DORN \\ Aircraft Gas Turbine Development Department, General Electric Company \\ AND \\ H. J. GREENBERG \\ New York University
}

1. Introduction. The variational principles of Greenberg and Prager [1] for the limit analysis of elastic-perfectly plastic structures require the optimization of a linear functional subject to linear constraints. The identification of these problems with linear programming problems was first made by Charnes and Greenberg [2]. In the present paper, the problems of limit analysis are reduced to three basic types of linear programming problems chosen so as to keep the size of the problem as small as possible. Appropriate methods of solution for each of these types of problems are reviewed and are adapted here for the special systems encountered. Procedures for determining an initial feasible solution for each of these methods, including a new procedure for determining an initial extreme point solution for use with Lemke's dual method [3], are discussed. The various linear programming solutions of the limit analysis problem are compared, and a simple example is carried through following each method of solution.

The structures considered are plane pin-jointed trusses for the sake of simplicity of description. The mathematical formulation would be the same, however, for rigid frames or continuous beams and for space as well as plane structures.

It should be emphasized that the real value of the linear programming solutions of limit analysis problems lies in the analysis of many variable problems arising in highly indeterminate structures. The methods of linear programming as outlined here lead to exact solutions by systematic procedures of types which are today routine for high speed digital computing machines.

Two codes for the solution of linear programming problems have been written for the IBM 701 computer $[10,11]$. Eisemann's code [10] employs the simplex technique as described in Sec. 4 below. The RAND code [11] is based on the "revised" simplex method which has certain advantages for high speed automatic computing. This latter code can accommodate 200 restraint equations. A structure with approximately 200 redundancies can be handled, therefore, using this code.

Codes have also been reported available for the UNIVAC and the IBM 650 computer [12].

2. Limit analysis problem. Consider a plane truss with no external redundancies and composed of $s$ bars and $k$ joints. Let a number of concentrated loads bearing fixed ratios and lying in the plane of the structure be applied at the joints of the truss. The equilibrium equations may then be written ${ }^{2}$

*Received May 11, 1956. This research was sponsored by the United States Air Force, through the Air Research and Development Command. The material contained in this paper was submitted as part of a thesis to the Carnegie Institute of Technology in May 1955, by the first author in partial fulfillment of the requirements for the degree of Doctor of Philosophy.

${ }^{1}$ Numbers in square brackets refer to the bibliography at the end of the paper.

2See for example, pp. 115-122 of Ref. [4]. 


$$
\sum_{i=1}^{\dot{a}} a_{i j} S_{i}=\lambda p_{i} \quad(i=1,2, \cdots, 2 k-3),
$$

where $S_{i}$ is the axial force in the $j$ th bar, considered positive for tensile forces; $\boldsymbol{p}_{i}$ are the fixed loads, and $\lambda$ is a positive multiplier which is allowed to increase monotonically from zero corresponding to proportional loading. The $a_{i j}$ depend on the geometrical configuration of the truss and are essentially direction cosines of the angles between the bars and the coordinate axes. If $s>2 k-3$ then the truss is redundant, and Eqs. (2.1) admit a non-trivial solution $S_{i}$ for $\lambda=0$. Indeed, if there are $r$ redundancies, then $s=2 k-$ $3+r$.

If it is assumed that the bars are composed of elastic-perfectly plastic material, the forces are further restricted by the yield conditions

$$
-L_{i} \leq S_{i} \leq U_{i} \quad(j=1,2, \cdots, s),
$$

where $U_{i}\left(-L_{i}\right)$ is the yield force in tension (compression).

By the maximum principle of limit analysis, the largest value of $\lambda$ for which a solution $S_{i}$, to Eqs. (2.1) and (2.2) exists, is the safety factor against plastic collapse [5].

3. The linear programming problem of Type I. Assuming, without loss of generality, that the first $2 k-3$ bars form a statically determinate truss, the equilibrium equations may be solved for the forces in the first $2 k-3$ bars in terms of the forces in the redundant members.

The maximum principle may then be phrased as follows in the standard notation and form of a linear programming problem.

Maximize

$$
\lambda=P_{0}^{\prime} x
$$

subject to

$$
P_{i}^{\prime} x \leq c_{i} \quad(j=1,2, \cdots, 28)
$$

where $x$ and $P_{i}(j=0,1, \cdots, 2 s)$ are column vectors ${ }^{3}$ in a real, $r+1$-dimensional vector space, $V_{r+1}$. The Cartesian components of these vectors are given by

$$
P_{i}=\left[\begin{array}{c}
-\sum_{i=1}^{2 k-3} a_{i i}^{-1} a_{i, 2 k-2} \\
-\sum_{i=1}^{2 k-3} a_{i i}^{-1} a_{i, 2 k-1} \\
-\sum_{i=1}^{2 k-3} a_{i i}^{-i} a_{i,} \\
+\sum_{i=1}^{2 k-3} a_{i i}^{-1} p_{i}
\end{array}\right] \quad(j=1, \cdots, 2 k-3)
$$
and $x$.

sPrime denotes transpose, i.e., a row vector, and $P_{0}^{b} x$, for example, denotes the inner product of $P_{0}$ 


$$
\begin{aligned}
P_{2 k-3+i}^{\prime} & =\left[\delta_{i 1}, \cdots, \delta_{j r}, 0\right] & (j=1,2, \cdots, r) \\
P_{i+。}^{\prime} & =-P_{i}^{\prime} & (j=1,2, \cdots, s) \\
P_{0}^{\prime} & =[0, \cdots, 0,1] & \\
x & =\left[S_{2 k-2}, \cdots, S_{s}, \lambda\right] &
\end{aligned}
$$

and the $c_{i}$ are real scalars defined by

$$
c_{i}= \begin{cases}U_{i} & (j=1,2, \cdots, s) \\ L_{i-s} & (j=s+1, \cdots, 2 s) .\end{cases}
$$

The $a_{i i}^{-1}$ are defined by

$$
\sum_{i=1}^{2 k-3} a_{i i}^{-1} a_{i m}=\delta_{i m} \quad(j, m=1,2, \cdots, 2 k-3),
$$

where $\delta_{i m}$ is the Kronecker delta."

Notice that

$$
c_{i} \geq 0 \quad(j=1,2, \cdots, 2 s) .
$$

The problem stated in (3.1) and (3.2) will be referred to as a linear programming problem of Type $I$ and is naturally adapted to solution by Lemke's Dual Method [3]. The fact that $P_{i+\varepsilon}=-P_{i}$, however, provides a simplification since only the first $s$ vectors need be carried in the calculations.

The tableau arrangement [6] developed by Orden, Dantzig, and Hoffman is modified by the addition of a final row with entries $-\left(z_{i+},-c_{i+\varepsilon}\right)$ as shown in Table I.

The vectors $a_{i}$ constitute a basis for $V_{r+1}$ chosen from among the $P_{i}$ so that $z_{j}-$ $c_{i} \leq 0$ for all $j, P_{k}^{\prime} \alpha_{a}$ is the component of $P_{k}$ along the vector $a_{a}$, and $z_{j}=\sum_{i=1}^{r+1}\left(P_{j}^{\prime} \alpha_{i}\right) c_{i}$. The last row is computed from

$$
-\left(z_{i+\ell}-c_{i+s}\right)=\left(z_{i}-c_{j}\right)+\left(U_{i}+L_{i}\right) .
$$

An optimum solution has been obtained if the elements in the $P_{0}$ column are nonnegative. If, however, $P_{0}^{\prime} \alpha_{a}<0$ then the negative entries in the $q$ th row are divided into the corresponding entries in the $z_{i}-c_{i}$ row, and the positive entries in the $q$ th row are divided into the corresponding entries in the $-\left(z_{j+\ell}-c_{i+\varepsilon}\right)$ row. The minimum of these quotients is selected. If this minimum occurs for $j=k$, there are two cases to be considered.

Case I: $P_{k}^{\prime} \alpha_{a}<0$. Then $P_{k}$ replaces $a_{a}$ in the basis and the tableau corresponding to the new basis is obtained from

$$
P_{i}=\sum_{\substack{i=1 \\ i \neq a}}^{r+1}\left[P_{j}^{\prime} \alpha_{i}-\frac{P_{j}^{\prime} \alpha_{a}}{P_{k}^{\prime} \alpha_{a}} P_{k}^{\prime} \alpha_{i}\right] a_{i}+\frac{P_{i}^{\prime} \alpha_{a}}{P_{k}^{\prime} \alpha_{a}} P_{k} .
$$

Note that the $a_{j i}^{-1}$ represent the elements of the inverse of the coefficient matrix of the unknown forces $S_{1}, \cdots, S_{2 k-3}$ in the equilibrium equations.

This permits these forces to be expressed in terms of the redundant forces in order to deal with the latter exclusively. However, in actual problems equilibrium equations which express each force explicitly in terms of the redundant forces can usually be written down directly from the structure without need of matrix inversion. 


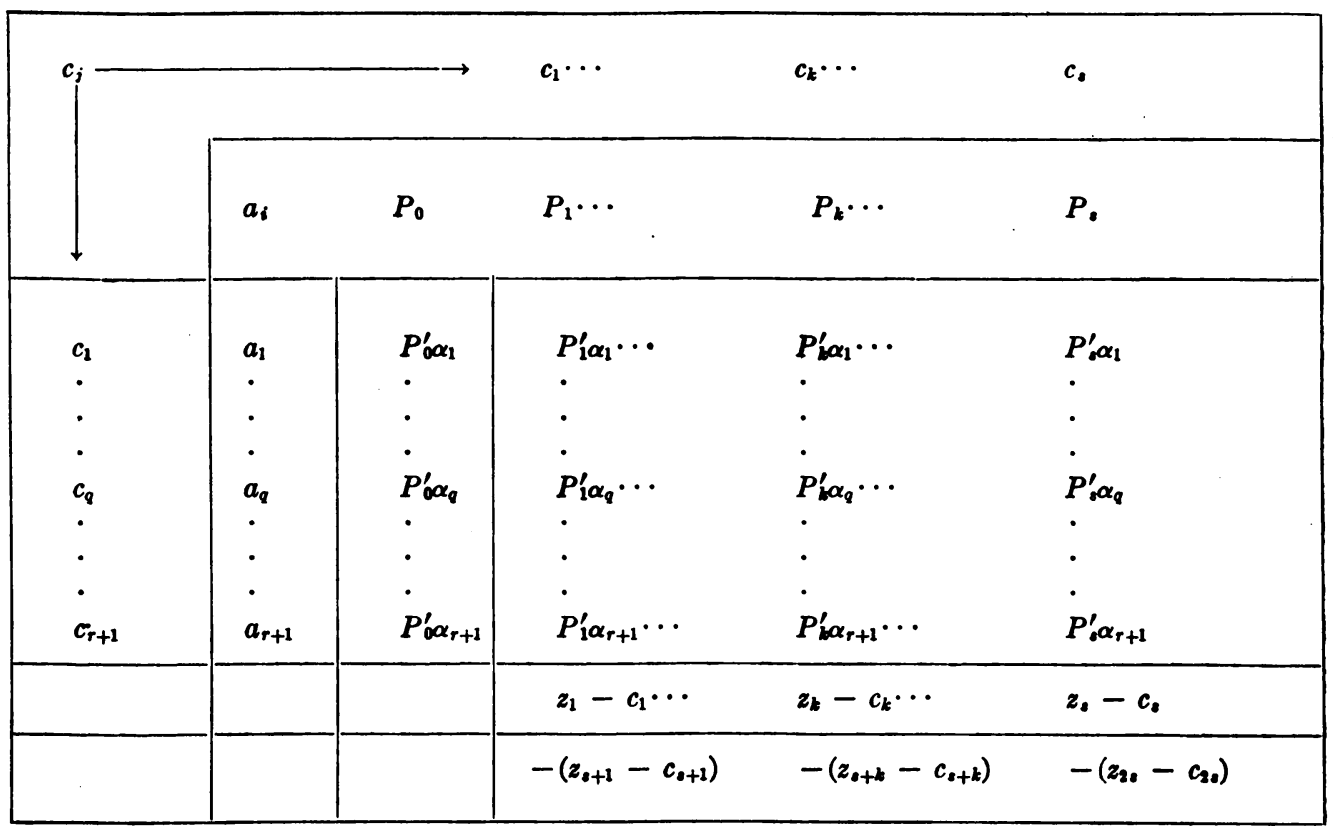

TABLE I

Case II: $P_{k}^{\prime} \alpha_{a}>0$. Then $P_{a+k}$ replaces $a_{a}$ in the basis and the new tableau is found from

$$
P_{i}=\sum_{\substack{i=1 \\ i \neq q}}^{r}\left[P_{i}^{\prime} \alpha_{i}-\frac{P_{j}^{\prime} \alpha_{q}}{P_{k}^{\prime} \alpha_{a}} P_{k}^{\prime} \alpha_{i}\right] a_{i}-\frac{P_{j}^{\prime} \alpha_{q}}{P_{k}^{\prime} \alpha_{a}} P_{k+a}
$$

When the optimum solution has been obtained, the values of the forces in the members are determined from

$$
S_{i}=\frac{1}{2}\left(z_{i}-c_{i}\right)-\left(z_{i+\iota}-c_{i+\_}\right)+\left(U_{i}-L_{i}\right) \quad(j=1,2, \cdots, 8) .
$$

4. Type II or simplex problem. The dual linear programming problem to the one defined by (3.1) and (3.2) is:

Minimize

$$
z_{0}=\sum_{i=1}^{2 s} \rho_{i} c_{i}
$$

over the variables $\rho_{i}$ subject to

$$
\sum_{i=1}^{20} \rho_{i} P_{i}=P_{0}
$$

and

$$
\rho_{i} \leq 0 \quad(j=1,2, \cdots, 2 s) .
$$

This will be referred to as a linear programming problem of Type II or Simplex Problem, and the dual theorem ${ }^{5}$ relating it to the Type I problem states that if either 
the minimum of $z_{0}$ or the maximum of $P_{0}^{\prime} x$ exists and is finite, then

$$
\text { Minimum } z_{0}=\text { Maximum } P_{0}^{\prime} x \text {. }
$$

The minimum problem phrased in (4.1), (4.2), (4.3) was shown to be equivalent to the kinematic principle of limit analysis by Charnes and Greenberg [2]. This problem is naturally adapted to solution by the simplex technique $[6,7]$.

The same tableau (Table I) as used in the dual method for the Type I problem may be used provided the basis vectors, $a_{i}$, are now chosen so that $P_{0}^{\prime} \alpha_{i} \geq 0$ for all $i$.

An optimum solution is at hand if $z_{j}-c_{j} \leq 0$ for all $j$. If $z_{k}-c_{k}>0$ then $P_{k}$ may be brought into the basis to obtain a smaller value of $z_{0}$. Two cases arise:

Case I: $0<k \leq s$. The positive entries in the $P_{k}$ column are divided into the corresponding entries in the $P_{0}$ column and the minimum of these quotients is selected. If the minimum occurs for $i=q$, then $P_{k}$ replaces $a_{q}$ in the basis and the algorithm for obtaining the entries for the new tableau is given by (3.6).

Case II: $s<k \leq 2 s$. The negative entries in the $P_{k}$ column are divided into the corresponding entries in the $P_{0}$ column, and the minimum of the absolute values of these quotients is selected. If the minimum occurs for $i=q$, then $P_{a+k}$ replaces $a_{q}$ in the basis and the algorithm for the new tableau is (3.7).

Again at the optimum solution the $S_{i}$ are determined by (3.8).

5. A bounded variables problem. The collapse problem stated in (2.1) and (2.2) may be transformed into still another type of linear programming problem which follows,

Maximize

$$
\lambda=\sum_{j=1}^{s+1} w_{j} c_{j}
$$

subject to

$$
\begin{gathered}
P_{0}=\sum_{i=1}^{+1} w_{i} P_{i} \\
0 \leq w_{i} \leq b_{i} \quad(j=1, \cdots, s+1),
\end{gathered}
$$

where $P_{i}(j=0,1, \cdots, s+1)$ are vectors in a space of $2 k-3$ dimensions, $V_{2 k-\mathbf{s}}$, and are defined as

$$
\begin{aligned}
& P_{0}=\left[\begin{array}{c}
\sum_{i=1}^{\dot{1}} a_{1 i} L_{i} \\
\sum_{i=1}^{\dot{1}} a_{2 j} L_{i} \\
\vdots \\
\sum_{i=1}^{\dot{a}} a_{2 k-3, i} L_{j}
\end{array}\right] \quad P_{i}=\left[\begin{array}{c}
a_{1 j} L_{i} \\
a_{2 j} L_{i} \\
\vdots \\
a_{2 k-3, j} L_{j}
\end{array}\right] \quad(j=1,2, \cdots, s) \\
& P_{a+1}=\left[\begin{array}{c}
-p_{1} \\
-p_{2} \\
\vdots \\
-p_{2 k-2}
\end{array}\right]
\end{aligned}
$$


and $w_{i}, c_{i}$ and $b_{i}$ are given by

$$
\begin{aligned}
& w_{i}= \begin{cases}\frac{S_{i}}{L_{i}}+1 & (j=1,2, \cdots, s) \\
\lambda & (j=s+1)\end{cases} \\
& c_{i}= \begin{cases}0 & (j=1,2, \cdots, s) \\
1 & (j=s+1)\end{cases} \\
& b_{i}= \begin{cases}\frac{U_{i}+L_{i}}{L_{i}} & (j=1,2, \cdots, s) \\
M & (j=s+1)\end{cases}
\end{aligned}
$$

where $M$ is an arbitrarily large positive number.

This can be then reduced to a Type II or simplex problem by introducing nonnegative variables $y_{i}$ such that

$$
w_{i}+y_{i}=b_{i} \text {. }
$$

This results in a Type II problem of size $(2 k+s-2) \times(2 s+2)$.

Charnes and Lemke [8] and Dantzig [9] have shown that this may be treated as a $(2 k-3) \times(s+1)$ problem, i.e., the inequalities $w_{i} \leq b_{i}$ may be suppressed.

6. Comparison of methods. A significant difference between the simplex and dual methods is that in the former a certain freedom of choice may be available in the vectors entering the basis, while in the dual method a choice may exist in the vector leaving the basis. For either method, therefore, a physical criterion is desirable to guide the analyst in his choice.

For the collapse problem, if at the optimum solution $P_{i}$ is in the basis for $j=1,2$, $\cdots, s$, the $j$ th bar yields in tension at collapse. Similarly if $P_{j}$ is present in the basis for $j=s+1, s+2, \cdots, 2 s$, then the $(j-s)$ th bar is yielding in compression.

Similar interpretations are available in the bounded variables formulation (Sec. 5).

These facts allow the experienced analyst to use his judgment and intuition in making choices regarding basis vectors.

A more quantitative comparison of the methods of solution arises from a consideration of the number of arithmetical operations necessary to achieve a solution.

As a measure of the arithmetical operations, the number of multiplications to be performed per iteration will be used. For Type I and II formulations this measure is $(r+2)(s+1)$, while for a bounded variables problem the number of multiplications is $(s-r+1)(s+2)$. The preference for formulation on the basis of the number of arithmetical operations depends, therefore, on the relationship between $s$, the number of bars, and $r$, the number of redundancies.

7. Initial solutions to the programming problems. In this section methods for obtaining initial feasible solutions to Type I and Type II linear programming problems are presented. For bounded variables problems it is sufficient to find a basis for the vector space of the equalities (5.2). The method developed for Type II problems produces such a basis.

A. Type I problems. If any point $x$ satisfying (3.2) can be found then a simple change of variables will translate this point to the origin. Starting from the origin, the following 
method then produces an extreme point solution (or initial feasible solution) by use of the dual method applied to a modified problem.

For the collapse problem, $c_{i} \geq 0$ and this implies that $x \equiv 0$ is a solution to (3.2).

Consider now the modified $^{6}$ problem to maximize $P_{0}^{\prime} x$ subject to

$$
\begin{aligned}
P_{i}^{\prime} x \leq c_{i} & (j=1,2, \cdots, 2 s) \\
\sigma_{i} e_{i}^{\prime} x \leq 0 & (i=1,2, \cdots, r+1) \\
c_{i} & \geq 0,
\end{aligned}
$$

where $e_{i}$ is a unit vector in $V_{r+1}$ with +1 as the $i$ th component and $\sigma_{i}$ is either equal to +1 or -1 and is chosen according to the criteria described below.

Notice that the origin is an extreme point of the modified problem regardless of the choice of sign for $\sigma_{i}$. The basis vectors associated with this point are $e_{i}$ for $i=1,2, \cdots$, $s+1$.

The set of points $x$ satisfying (7.1) is designated by $\Lambda$. The set satisfying both (7.1) and (7.2) is $\Lambda^{*}$. Note that (7.2) is just a restriction to some orthant of $V_{r+1}$ once the signs of $\sigma_{i}$ have been chosen. In order that the solution to the modified problem coincide with the original problem, it is necessary and sufficient that the $\sigma_{i}$ be chosen in (7.2) so that the point $x$ in $\Lambda$ for which $P_{0}^{\prime} x$ takes on its maximum also is contained in $\Lambda^{*}$, i.e., the correct orthant of $V_{r+1}$ must be chosen.

The advantage of the modified problem is, of course, that $x \equiv 0$ is an extreme point solution. Starting from this solution and using the dual method, a value of $\sigma_{i}$ for some $i$ is chosen at each iteration, and the corresponding $e_{i}$ leaves the basis in favor of some $P_{i}$.

The procedure for accomplishing this is as follows. Let the basis at some stage be $e_{1}, \cdots, e_{t}, P_{t+1}, \cdots, P_{r+1}$ where the signs of $\sigma_{t+1}, \cdots, \sigma_{r+1}$ have already been properly chosen.

Define $r+1$ vectors $\alpha_{i}(j=1,2, \cdots, r+1)$ such that

$$
e_{i}^{\prime} \alpha_{j}=\left\{\begin{array}{lll}
1 & \text { if } & i=j \\
0 & \text { if } & i \neq j
\end{array}\right.
$$

for $i=1,2, \cdots, t$ and

$$
P_{i}^{\prime} \alpha_{i}=\left\{\begin{array}{lll}
1 & \text { if } & i=j \\
0 & \text { if } & i \neq j
\end{array}\right.
$$

for $i=t+1, \cdots, r+1$.

Then

$$
P_{0}=\sum_{i=1}^{t}\left(P_{0}^{\prime} \alpha_{i}\right) e_{i}+\sum_{i=t+1}^{r+1}\left(P_{0}^{\prime} \alpha_{i}\right) P_{i}
$$

and if $x_{0}$ is the extreme point of $A^{*}$ corresponding to this basis then by definition

$$
\begin{aligned}
e_{i}^{\prime} x_{0}=0 & (i=1,2, \cdots, t) \\
P_{i}^{\prime} x_{0}=c_{i} & (i=t+1, \cdots, r+1) .
\end{aligned}
$$

'The authors are indebted to Dr. C. E. Lemke for suggesting this modification. 
Let

$$
\bar{x}=x_{0}-\theta \alpha_{a} \quad 1 \leq q \leq t
$$

then

$$
\begin{aligned}
P_{0}^{\prime} \bar{x}=\sum_{\substack{i=1 \\
i \neq a}}^{t}\left(P_{o}^{\prime} \alpha_{i}\right)\left(e_{i}^{\prime} x_{0}\right)-\theta \sum_{\substack{i=1 \\
i=a}}^{t}\left(P_{0}^{\prime} \alpha_{i}\right)\left(e_{i}^{\prime} \alpha_{q}\right)+ & \left(P_{o}^{\prime} \alpha_{q}\right)\left(e_{q}^{\prime} \bar{x}\right) \\
& +\sum_{i=t+1}^{r+1}\left(P_{0}^{\prime} \alpha_{i}\right)\left(P_{i}^{\prime} x_{0}\right)-\theta \sum_{i=t+1}^{r+1}\left(P_{0}^{\prime} \alpha_{i}\right)\left(P_{i}^{\prime} \alpha_{q}\right) .
\end{aligned}
$$

Now from (7.4) and (7.5)

$$
\begin{aligned}
e_{i}^{\prime} \alpha_{a}=0 & (i=1, \cdots, q-1, q+1, \cdots, t) \\
P_{i}^{\prime} \alpha_{q}=0 & (i=t+1, \cdots, r+1)
\end{aligned}
$$

and using (7.6)

$$
P_{0}^{\prime} \bar{x}=\left(P_{0}^{\prime} \alpha_{q}\right)\left(e_{q}^{\prime} \bar{x}\right)+\sum_{i=t+1}^{r+1}\left(P_{0}^{\prime} \alpha_{i}\right)\left(P_{i}^{\prime} x_{0}\right) .
$$

The last sum is $P_{0}^{\prime} x_{0}$ so

$$
P_{0}^{\prime} \bar{x}=P_{0}^{\prime} x_{0}+\left(P_{0}^{\prime} \alpha_{a}\right)\left(e_{a}^{\prime} \bar{x}\right) .
$$

Three cases arise: (i) $P_{0}^{\prime} \alpha_{a}<0$; (ii) $P_{0}^{\prime} \alpha_{a}>0$; (iii) $P_{0}^{\prime} \alpha_{q}=0$.

For case (i) if $e_{a}^{\prime} \bar{x}>0$ then $P_{0}^{\prime} \bar{x}<P_{0}^{\prime} x_{0}$. It follows that any $\bar{x}$ yielding a larger value of the functional than $x_{0}$ cannot lie in the half-space $e_{q}^{\prime} \bar{x}>0$, but must satisfy

$$
+e_{a}^{\prime} \bar{x} \leq 0 .
$$

Thus $e_{a}$ is removed from the basis and we pick $\sigma_{q}=+1$.

For case (ii) if $e_{a}^{\prime} \bar{x}<0$ then again $P_{0}^{\prime} \bar{x}<P_{0}^{\prime} x_{0}$. Thus similarly it is necessary that

$$
-e_{\sigma}^{\prime} \bar{x} \leq 0 \text {. }
$$

Again $e_{a}$ is removed from the basis but $\sigma_{a}$ is chosen to be -1 .

Finally for case (iii), $P_{0}^{\prime} \bar{x}=P_{0}^{\prime} x_{0}$ and the choice of $\sigma_{u}$ is deferred for the present.

If at some stage of the computations, case (iii) holds for all $e_{i}$ remaining in the basis then the choice of $\sigma_{i}$ for those $e_{i}$ is arbitrary.

The usual algorithms (3.6) and (3.7) are used to proceed to a new tableau.

In this way in $r+1$ iterations all of the $e_{i}$ are eliminated from the basis and the appropriate values of $\sigma_{i}$ equal to +1 or -1 are chosen.

B. Type II problems. Consider the following modification ${ }^{7}$ of the Type II problem formulated in (4.1), (4.2), (4.3): To minimize

subject to

$$
z_{0}=\sum_{i=1}^{28} \rho_{i} c_{i}+\sum_{i=1}^{r+1} \gamma_{i} M
$$

$$
P_{0}=\sum_{i=1}^{2 s} \rho_{i} P_{i}+\sum_{i=1}^{r+1} \gamma_{i} e_{i}
$$

This modification was first suggested by Dantzig [7], see footnote to p. 340. This is the technique employed in the 701 codes $[10,11]$ to obtain initial solutions. 


$$
\begin{array}{ll}
\rho_{i} \geq 0 & (j=1,2, \cdots, 2 s) \\
\gamma_{i} \geq 0 & (i=1,2, \cdots, r+1),
\end{array}
$$

where $e_{i}$ is a unit vector with plus or minus one as the $i$ th component depending on whether the $i$ th component of $P_{0}$ is positive or negative, and where $M$ is an arbitrarily large positive number.

The solution to this problem is identical with the one phrased in (4.1), (4.2), (4.3) since the minimum will occur for $\gamma_{i}=0$ for all $i$.

A basis for this problem, however, is readily available. Indeed, the basis $a_{i}$ may be taken to be

$$
a_{i}=e_{i} \quad(i=1,2, \cdots, r+1) .
$$

It follows from the definitions of $e_{i}$ and $\alpha_{i}$ that

$$
\alpha_{i}=e_{i} \quad(i=1,2, \cdots, r+1) .
$$

The entries of the tableau (Table I) are, therefore, easily computed.

The vectors $e_{i}$ need not be carried in the tableau since if an $e_{i}$ leaves the basis it cannot return because it carries a large positive weight, $M$. It will require exactly $r+1$ iterations in order to obtain a basis comprised entirely of vectors chosen from among the $P_{i}$.

8. Examples. To illustrate and compare the methods of solution of linear programming problems described in the previous sections as applied to structural collapse problems, a simple example and its solution by these methods are presented here.

Consider the once redundant truss in Fig. 1 loaded with a single concentrated force

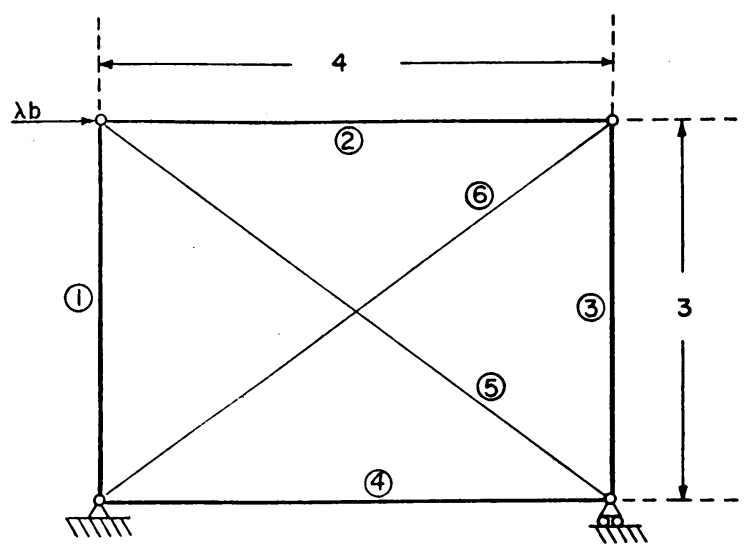

FIG. 1.

as shown. The members are numbered as indicated, and the equilibrium equations may be written

$$
\begin{aligned}
-S_{2}-\frac{4}{5} S_{5} & =\lambda b \\
S_{2}+\frac{4}{5} S_{6} & =0 \\
S_{4}+\frac{4}{5} S_{5} & =0 \\
S_{1}+\frac{3}{5} S_{5} & =0 \\
S_{3}+\frac{3}{5} S_{6} & =0
\end{aligned}
$$


The fully plastic forces in tension and compression are taken to be the same and equal to $N_{p}$. The yield criteria (2.1) become then

$$
\left|S_{i}\right| \leq N_{p} \quad(i=1,2, \cdots, 6) .
$$

Now letting $x_{1}=S_{6} / N_{p}$ and $x_{2}=\lambda b / N_{p}$ and solving the equilibrium equations for $S_{1}, S_{2}, \cdots, S_{5}$ in terms of these, the yield criteria may be written

$$
\begin{aligned}
\left|-\frac{3}{5} x_{1}+\frac{3}{4} x_{2}\right| & \leq 1, \\
\left|-\frac{4}{5} x_{1}\right| & \leq 1, \\
\left|-\frac{3}{5} x_{1}\right| & \leq 1, \\
\left|-\frac{4}{5} x_{1}+x_{2}\right| & \leq 1, \\
\left|x_{1}-\frac{5}{4} x_{2}\right| & \leq 1, \\
\left|x_{1}\right| & \leq 1 .
\end{aligned}
$$

The safety factor against collapse is the largest value of $x_{2} N_{p} / b$ consistent with the above inequalities.

Define 13 vectors in a two-dimensional space as

$$
\begin{gathered}
P_{1}^{\prime}=-P_{7}^{\prime}=\left(-\frac{3}{5}, \frac{3}{4}\right), \quad P_{2}^{\prime}=-P_{8}^{\prime}=\left(-\frac{4}{5}, 0\right), \\
P_{3}^{\prime}=-P_{9}^{\prime}=\left(-\frac{3}{5}, 0\right), \quad P_{4}^{\prime}=-P_{10}^{\prime}=\left(-\frac{4}{5}, 1\right), \\
P_{5}^{\prime}=-P_{11}^{\prime}=\left(1,-\frac{5}{4}\right), \quad P_{6}^{\prime}=-P_{12}^{\prime}=(1,0), \\
P_{0}^{\prime}=(0,1) .
\end{gathered}
$$

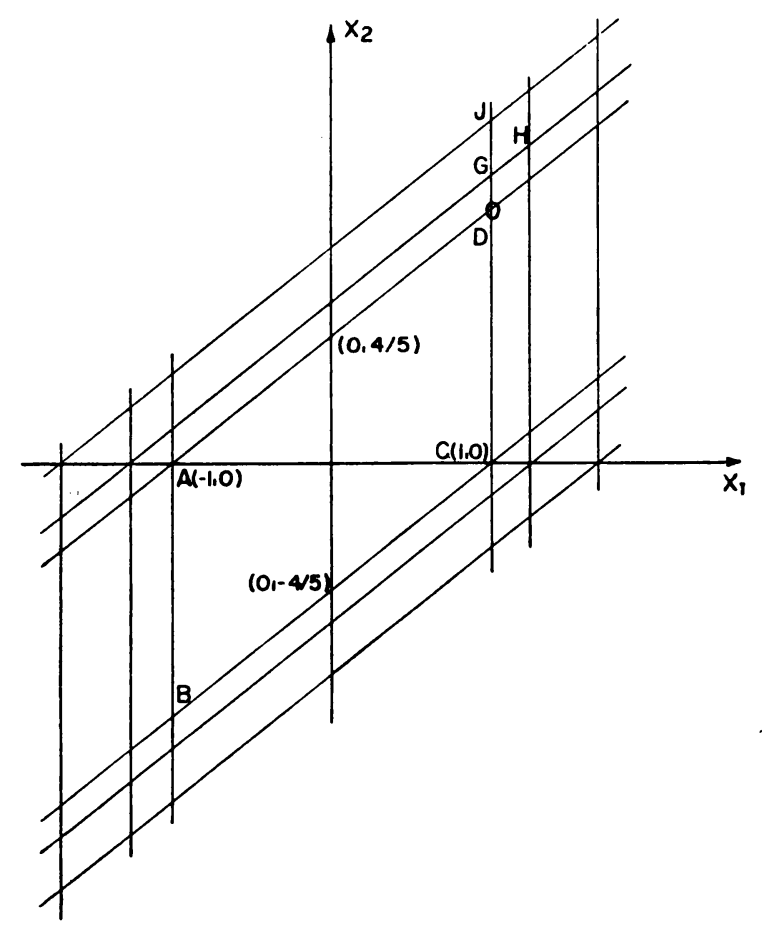

Fig. 2. 
The problem in vector notation is then to maximize $x_{2}=P_{0}^{\prime} x$ subject to

$$
P_{i}^{\prime} x \leq 1 \quad(j=1,2, \cdots, 12) .
$$

The equation $P_{i}^{\prime} x=1$ defines a line in two-space for each $j$. The vector $P_{i}$ is normal to this line and points into the half-space for which the corresponding inequality is violated. All of the lines defined by equality in (8.1) are shown in Fig. 2. The set of points $\Lambda$ for which all of (8.1) are satisfied in the parallelogram $A B C D$, and the maximum value of $x_{2}$ is obtained at the point $D(1,8 / 5)$. The safety factor is then

$$
\lambda=\frac{8}{5} \frac{N_{p}}{b} .
$$

An analytic solution is obtained by the dual method. Let

$$
e_{1}=\left(\begin{array}{l}
1 \\
0
\end{array}\right) \quad e_{2}=\left(\begin{array}{l}
0 \\
1
\end{array}\right)
$$

be the initial basis for the space. This is not an extreme point solution of $\Lambda$ but is used here to find such an extreme point solution. The initial tableau appears in Table IIA.

Since $P_{0}^{\prime} \alpha_{2}>0, \sigma_{2}$ is chosen to be -1 . This multiplies the $e_{2}$ row by -1 . Now choose the minimum over $j$ of

$$
\begin{gathered}
\frac{z\left(P_{i}\right)}{P_{i}^{\prime} \alpha_{2}} \text { for } P_{i}^{\prime} \alpha_{2}<0, \\
\frac{-z\left(P_{i+8}\right)}{P_{i}^{\prime} \alpha_{2}} \text { for } \quad P_{i}^{\prime} \alpha_{2}>0 .
\end{gathered}
$$

This occurs for $j=5$ and is $4 / 5$. Since $P_{5}^{\prime} \alpha_{2}>0, P_{11}$ replaces $e_{2}$ in the basis.

The result is Table IIB. The process is iterated and $\sigma_{1}$ is chosen to be -1 after which $P_{6}$ replaces $e_{1}$. Table IIC represents the final solution.

The maximum value of $x_{2}, 8 / 5$, appears in the $P_{0}$ column and the $z\left(P_{i}\right)$ row. Thus $\lambda=8 N_{p} / 5 b$. The presence of $P_{6}$ and $P_{11}$ in the basis indicates that bar 6 yields in tension and bar 5 in compression, i.e., $S_{5}=-N_{p}, S_{6}=N_{p}$. Because of the normalization used here, Eq. (3.8) for the $S_{i}$ is

$$
S_{i}=\frac{1}{2}\left[\left(z_{i}-c_{i}\right)-\left(z_{i+。}-c_{i+\curvearrowleft}\right)\right] N_{p} .
$$

Thus

$$
S_{1}=3 N_{p} / 5, \quad S_{2}=-4 N_{p} / 5, \quad S_{3}=-3 N_{p} / 5, \quad S_{4}=4 N_{p} / 5 .
$$

The dual to this problem is a simplex problem and the same initial tableau (Table IIA) may be used if the vectors $e_{1}, e_{2}$ are given large positive weights, $M$. Now many vectors, i.e. those for which $z\left(P_{j}\right)>0$, may enter the basis. $P_{6}$ is chosen on an intuitive basis and thus $e_{1}$ leaves the basis. The resulting tableau is shown in Table IIIA. $P_{4}$ then replaces $e_{2}$ and Table IIIB results. Finally $P_{11}$ replaces $P_{4}$ and the final tableau will be identical with Table IIC.

Notice that the solution in Table IIIA corresponds to the point $(1, M)$ and the solution in Table IIIB to the point $G(1,9 / 5)$ in Fig. 2.

Finally, the problem can be formulated as a bounded variables problem of size $(5 \times 7)$. 


\begin{tabular}{|c|c|c|c|c|c|c|c|c|}
\hline \multicolumn{3}{|c|}{$C_{j} \longrightarrow$} & 1 & 1 & 1 & 1 & 1 & 1 \\
\hline & $a_{i}$ & $P_{0}$ & $P_{1}$ & $P_{2}$ & $P_{3}$ & $P_{4}$ & $P_{5}$ & $P_{6}$ \\
\hline 0 & $e_{i}$ & 0 & $-3 / 5$ & $-4 / 5$ & $-3 / 5$ & $-4 / 5$ & 1 & 1 \\
\hline $\mathbf{0}$ & $e_{2}$ & 1 & $3 / 4$ & 0 & $\mathbf{0}$ & 1 & $-5 / 4$ & $\mathbf{0}$ \\
\hline \multicolumn{2}{|c|}{$Z\left(P_{j}\right)$} & 0 & -1 & -1 & -1 & -1 & -1 & -1 \\
\hline \multicolumn{3}{|c|}{$-Z\left(P_{j+6}\right) \longrightarrow$} & +1 & +1 & +1 & +1 & $t$ & +1 \\
\hline
\end{tabular}

\begin{tabular}{|c|c|c|c|c|c|c|c|c|}
\hline \multicolumn{3}{|c|}{$C_{i} \longrightarrow$} & 1 & 1 & 1 & 1 & 1 & 1 \\
\hline & $a_{i}$ & $P_{0}$ & $P_{1}$ & $P_{2}$ & $P_{3}$ & $P_{4}$ & $P_{5}$ & $P_{6}$ \\
\hline 0 & $e_{1}$ & $4 / 5$ & $\mathbf{0}$ & $-4 / 5$ & $-3 / 5$ & 0 & $\mathbf{0}$ & 1 \\
\hline 1 & $P_{11}$ & $4 / 5$ & $3 / 5$ & 0 & $\mathbf{0}$ & $4 / 5$ & -1 & 0 \\
\hline \multicolumn{2}{|c|}{$Z\left(P_{i}\right)$} & $4 / 5$ & $-2 / 5$ & -1 & -1 & $-1 / 5$ & -2 & -1) \\
\hline \multicolumn{3}{|c|}{$-Z\left(P_{j+6}\right) \longrightarrow \longrightarrow$} & $+8 / 5$ & +1 & +1 & $+9 / 5$ & 0 & +1 \\
\hline
\end{tabular}

\begin{tabular}{|c|c|c|c|c|c|c|c|c|}
\hline \multicolumn{3}{|c|}{$C_{i} \longrightarrow \longrightarrow$} & 1 & 1 & 1 & 1 & 1 & 1 \\
\hline$\downarrow$ & $a_{i}$ & $P_{0}$ & $P_{1}$ & $P_{2}$ & $P_{3}$ & $P_{4}$ & $P_{5}$ & $P_{6}$ \\
\hline 1 & $P_{6}$ & $4 / 5$ & 0 & $-4 / 5$ & $-3 / 5$ & $\mathbf{0}$ & $\mathbf{0}$ & 1 \\
\hline 1 & $P_{11}$ & $4 / 5$ & $3 / 5$ & $\mathbf{0}$ & $\mathbf{0}$ & $4 / 5$ & -1 & 0 \\
\hline \multicolumn{2}{|c|}{$Z\left(P_{j}\right)$} & $8 / 5$ & $-2 / 5$ & $-9 / 5$ & $-8 / 5$ & $-1 / 5$ & -2 & 0 \\
\hline \multicolumn{3}{|c|}{$-Z\left(P_{j+6}\right) \longrightarrow \longrightarrow$} & $+8 / 5$ & $+1 / 5$ & $+2 / 5$ & $+9 / 5$ & $\mathbf{0}$ & +2 \\
\hline
\end{tabular}

TABLE II.

Dual method solution.

\section{BIBLIOGRAPHY}

1. H. J. Greenberg and W. Prager, Limit design of beams and frames, Proc. ASCE 77, Sep. No. 59, (Feb. 1951)

2. A. Charnes and H. J. Greenberg, Plastic collapse and linear programming, abstract presented at Summer Meeting of Amer. Math. Soc., Sept. 1951 


\begin{tabular}{|c|c|c|c|c|c|c|c|c|}
\hline \multicolumn{3}{|c|}{$C_{j} \longrightarrow$} & 1 & 1 & 1 & 1 & 1 & 1 \\
\hline$\downarrow$ & $a_{\mathbf{i}}$ & $P_{0}$ & $P_{1}$ & $P_{2}$ & $P_{3}$ & $P_{4}$ & $P_{5}$ & $P_{6}$ \\
\hline 1 & $P_{6}$ & $\mathbf{0}$ & $-3 / 5$ & $-4 / 5$ & $-3 / 5$ & $-4 / 5$ & 1 & 1 \\
\hline$M$ & $e_{2}$ & 1 & $3 / 4$ & $\mathbf{0}$ & $\mathbf{0}$ & 1 & $-5 / 4$ & $\mathbf{0}$ \\
\hline $\boldsymbol{Z}\left(P_{i}\right)$ & & $M$ & $\begin{array}{r}3 M / 4 \\
-8 / 5\end{array}$ & $-9 / 5$ & $-8 / 5$ & $M-9 / 5$ & $-5 M / 4$ & $\mathbf{0}$ \\
\hline \multicolumn{3}{|c|}{$-Z\left(P_{j+8}\right) \longrightarrow \longrightarrow$} & $\begin{array}{l}3 M / 4 \\
\quad+2 / 5\end{array}$ & $+1 / 5$ & $+2 / 5$ & $M+1 / 5$ & $\begin{array}{c}-5 M / 4 \\
+2\end{array}$ & 2 \\
\hline
\end{tabular}

\begin{tabular}{|c|c|c|c|c|c|c|c|c|}
\hline \multicolumn{3}{|c|}{$C_{j} \longrightarrow$} & 1 & 1 & 1 & 1 & 1 & 1 \\
\hline 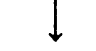 & $a_{i}$ & $P_{0}$ & $P_{1}$ & $P_{2}$ & $P_{3}$ & $P_{4}$ & $P_{5}$ & $P_{6}$ \\
\hline 1 & $P_{6}$ & $4 / 5$ & 0 & $-4 / 5$ & $-3 / 5$ & $\mathbf{0}$ & $\mathbf{0}$ & 1 \\
\hline 1 & $P_{4}$ & 1 & $3 / 4$ & 0 & 0 & 1 & $-5 / 4$ & 0 \\
\hline$Z\left(P_{j}\right)$ & & $9 / 5$ & $-1 / 4$ & $-9 / 5$ & $-8 / 5$ & $\mathbf{0}$ & $-9 / 4$ & 0 \\
\hline \multicolumn{3}{|c|}{$-Z\left(P_{j+6}\right) \longrightarrow \longrightarrow$} & $+7 / 4$ & $+1 / 5$ & $+2 / 5$ & +2 & $-1 / 4$ & +2 \\
\hline
\end{tabular}

TABLE III.

Simplex method solution.

3. C. E. Lemke, The dual method of solving the linear programming problem, Naval Research Logistics Quart. 1, No. 1, 36-47 (March 1954)

4. J. Nielsen, Vorlesungen über elementare Mechanik, Julius Springer, Berlin, 1935

5. H. J. Greenberg, paper presented at Second Symposium on Plasticity, Brown University, April 1949

6. A. Charnes, W. Cooper, and A. Henderson, Introduction to linear programming, John Wiley and Sons, 1953

7. G. B. Dantzig, "Maximization of a linear function of variables subject to linear inequalities", Chapter XXI of Activity analysis of production and allocation, Cowles Commission Monograph 13, John Wiley and Sons, 1951

8. A. Charnes and C. E. Lemke, Computational theory of linear programming I: The "Bounded variables" problem, ONR Research memorandum No. 10, Grad. School of Ind. Adm., Carnegie Inst. of Tech., Jan. 1954

9. George B. Dantzig, Upper bounds, secondary constraints, and block triangularity in linear programming, Econometrica 23, No. 2, 174-183 (April 1955)

10. K. Eisemann, Linear programming, Quart. Appl. Math. 13, No. 13, 209-232 (Oct. 1955)

11. William Orchard-Hays, The RAND code for the simplex method (SX4) (For the IBM 701 electronic computer), The RAND Corp., Research Memo. RM-1440 (7 Feb. 1955)

12. L. Wheaton Smith, Jr., Current status of the industrial use of linear programming, Management Science 2, No. 2, 156-158 (Jan. 1956) 